\title{
Psychological morbidity and resilience associated with tinnitus in a Sri Lankan population
}

\author{
T Abayabandara-Herath, A Rodrigo
}

\section{Abstract \\ Objective}

Tinnitus is a common and debilitating health condition. It is often associated with psychiatric morbidity. This important health concern has not been studied previously in Sri Lanka. We aimed to determine the prevalence of psychological morbidity, resilience and their correlates among a cohort of Sri Lankan patients with tinnitus.

\section{Methods}

A descriptive cross-sectional study was conducted among attendees of otolaryngology clinics in three Sri Lankan hospitals, using a specially designed questionnaire. Resilience was defined as absence of psychological morbidity and functional impairment.

Results

Of the 200 patients included in the study, $82.5 \%$ reported functional impairment due to tinnitus. The majority of participants were found to have depression (61.5\%). Sleep disturbance, feeling exhausted, loudness of tinnitus and functional impairment were significantly associated with psychological morbidity. Only $12 \%$ of participants were resilient and it was associated with mind-fulness and ability to distract self.

\section{Conclusions}

Tinnitus is a distressing illness associated with a significant functional impairment and psychiatric morbidity. We discuss the implication of this study for clinical practice.

Key words: tinnitus, psychological morbidity, resilience, Sri Lanka

SL J Psychiatry 2021; 12(2): 10-14

\section{Introduction}

Tinnitus is a perception of sound which is unrelated to an external acoustic source. It is usually described as a ringing noise. Tinnitus is a common health issue with a lifetime prevalence of $5.1 \%-42.7 \%$ (1). It adversely influences quality of life in affected individuals from a minor irritation to severe distress leading to self-harm (2). Tinnitus often presents with impaired functioning and psychiatric illnesses. Psychiatric diseases are highly prevalent among people with tinnitus, and $77 \%$ of population with tinnitus report a psychiatric disorder $(3,4)$. Post-traumatic stress disorder, insomnia, anxiety disorder and depressive disorder co-occur with tinnitus and the last two disorders are the most common comorbidities (5). These disorders will not only add to the burden of tinnitus, also exacerbate tinnitus. For example, studies have found that emotional exhaustion is a strong predictor of the severity of tinnitus (6). Therefore, the association between tinnitus and psychiatric disorders is bidirectional (7).
Although there are several treatment options, none of these interventions have been proven to consistently eliminate tinnitus (8). Further, most of these treatment modalities are not available in many regions in the world, including Sri Lanka. Therefore, it is of paramount importance to address treatable comorbidities, which in turn will improve the severity and impact of tinnitus. While treating psychiatric comorbidities is important, preventing such illnesses in tinnitus is ideal. Some people are resilient even in the face of debilitating illness such as tinnitus and adapt well without developing psychiatric illness. Wallhausser - Franke et al., who studied the association between tinnitus and resilience using the resilience scale, reported that loudness of the tinnitus, depression, anxiety, and somatic symptoms had an inverse correlation with resilience (3). The aims of the present study were to assess the prevalence, correlates of functional impairment and psychiatric morbidity in a cohort of people with tinnitus in Sri Lanka. Factors associated with resilience in this population were also explored. 


\section{Methodology}

This was a multi-centre cross sectional descriptive study conducted among Sri Lankan patients with the complaint of tinnitus. All eligible consenting consecutive adult patients who attended otolaryngology clinics in three large hospitals, namely Colombo South Teaching Hospital and Kandy Teaching Hospital, and District General Hospital, Matale, between August 2016 and April 2017 were recruited for the study. Patients with a diagnosis of mental illness prior to the onset of tinnitus were excluded from the study. Information was gathered using a self-administered questionnaire, which was distributed among the patients while they were waiting to be seen at the clinic. Participants were assisted to fill the questionnaire if they had difficulty in doing so and the questionnaires were collected upon completion.

Ethics clearance was granted from the Ethics Review Committee of the Faculty of Medicine, University of Kelaniya, Ragama and permission was obtained from relevant hospital authorities. Informed written consent was obtained from all participants.

\section{Data collection instruments}

Demographic and clinical characteristics were gathered using a specially designed pilot-tested questionnaire, which contained questions on participant's age, gender, religion, clinical characteristics of tinnitus, functional impairment due to tinnitus, associated factors and potential determinants of resilience based on the Resilience Scale.

The validated Sinhalese version of 12 - item General Health Questionnaire (GHQ-12) and Beck Depression Inventory-- II (BDI II) were used to detect psychiatric caseness and depression respectively. The GHQ - 12 is widely used to detect minor psychiatric disorders and BDI II is the most commonly used self-rating depression screening tool. Sinhala versions of these two scales have good psychometric properties $(9,10)$. Some studies have defined resilience as the absence of psychopathology following adversity (7). We defined resilience as the absence of psychiatric illness and functional impairment, for the purpose of this study $(11,12)$.

\section{Results}

Of the 200 patients who returned completed questionnaires, 124 were females (62\%). The average age of participants was 53.6 years $(S D \pm 14.6$, Range $=18$ to 75 years) and the average duration of tinnitus was 2.9 years. Participants' clinical characteristics of tinnitus are summarised in Table 1.

Only 14 (7\%) participants reported a history of severe noise exposure. A majority (51.5\%) of the patients had comorbid medical conditions such as diabetes mellitus, hypertension or dyslipidaemia. A majority (82.5\%) reported a functional impairment in one of the domains assessed (Table 2).

\begin{tabular}{|l|l|l|}
\hline \multicolumn{2}{|c|}{ Table 1. Clinical characteristics of tinnitus } \\
\hline Tinnitus Characteristics & No. & Percentage \% \\
\hline Site of tinnitus & & 27.5 \\
Bilateral & 55 & 32.5 \\
Right ear & 65 & 35.5 \\
Left ear & 71 & 4.5 \\
Head & 09 & 37.5 \\
\hline Onset of tinnitus & & 62.5 \\
Gradual & & \\
Sudden & 75 & 5 \\
\hline Type of tinnitus sound & 125 & 8 \\
Chirping & 10 & 65.5 \\
Pulsatile & 16 & 19.5 \\
Roaring & 131 & 2 \\
Whistling & 39 & \\
Other & 4 & \\
& & \\
\hline
\end{tabular}




\begin{tabular}{|l|l|l|}
\hline Tinnitus Characteristics & No. & Percentage \% \\
\hline Subjectively perceived loudness of & & 5.5 \\
tinnitus & & 8 \\
Minimal & 11 & 36 \\
Mild & 16 & 40.5 \\
Moderate & 72 & 10 \\
Severe & 81 & \\
Profound & 20 & 70.5 \\
\hline Nature of tinnitus & & 29.5 \\
Continuous & 141 & 41.5 \\
Intermittent & 59 & \\
Predominantly at night & 83 & 52 \\
\hline Associated problems & & 29 \\
Hearing loss & 104 & 53.5 \\
Ear pain & 58 & \\
Vertigo or giddiness & 107 & \\
\hline
\end{tabular}

\begin{tabular}{|c|c|c|}
\hline Impairment & Number & Percentage \% \\
\hline Difficulty in engaging in occupational work & 127 & 63.5 \\
\hline Difficulty in communicating with others & 118 & 59 \\
\hline Difficulty in sleeping & 135 & 67.5 \\
\hline Difficulty in completing daily household chores & 132 & 66 \\
\hline Difficulty in engaging in entertainment activities & 111 & 55.5 \\
\hline
\end{tabular}

One hundred and sixteen (68\%) participants scored above the cut-off of the GHQ 12, suggesting psychiatric casesness, and $61.5 \%$ were detected to have depression as per BDI II. There was a high correlation between these two measures with Pearson correlation coefficient $(\mathrm{r}=0.515)$. Sleep disturbance $(\mathrm{r}=0.426, \mathrm{p}<0.001)$, feeling exhausted $(r=0.390, p=0.003)$, uncontrollable nature of tinnitus $(\mathrm{r}=0.329, \mathrm{p}<0.001)$ and functional impairment $(r=0.231, p=0.001)$ were significantly associated with psychiatric comorbidity.

Resilience was defined as absence of psychiatric morbidity and functional impairment, and $12 \%$ of the participants were found to be resilient. A logistic regression analysis demonstrated that being able to distract self $(\mathrm{p}=0.017)$ and practicing mindfulness $(p=0.044)$ were significantly associated with resilience. However, being optimistic $(\mathrm{p}=0.09)$, being religious $(\mathrm{p}=0.2)$, appreciating the full context of any situation ( $\mathrm{p}$ $=0.1$ ), and perceived self-efficacy $(p=0.3)$ did not have significant associations with resilience.

\section{Discussion}

In this study, the first of its kind in Sri Lanka, we found psychological comorbidity and functional impairment to be common in patients with tinnitus. The demographic profile of our patients is largely similar to that of studies done elsewhere $(5,13,14)$. Previous studies have found that tinnitus is more prevalent in older ages, with a peak at 60 years (1). We too found that most of the patients with tinnitus were more than 50 years old. This has a clear implication for Sri Lanka, indicating that with a rapidly ageing population, the prevalence of tinnitus will increase in the future.

A majority of our study population reported significant impairment in all the functional domains we assessed. The disability or functional impairment in our study population is higher than what has been reported in previous studies (15). While direct comparisons of results are not possible due to differences in study population and methodology, lack of effective treatment, absence 
of patient support groups and poor public awareness that may have led to higher rates of reported disability in Sri Lanka.

The prevalence of depression in our study population is comparable to other similar studies. However, the prevalence of depression in this study population was almost ten times higher than the point prevalence in normative Sri Lankan population (16). Associated disability and lack of effective treatment for tinnitus in Sri Lanka may explain the high prevalence of depression. The rate of psychological distress or depression in our study is equivalent or higher than that of patients with lung cancers (67\%) and Parkinsons disease (48\%) in Sri Lanka $(17,18)$. The high prelavance of depression demonstrates the significant psychological impact of tinnitus, which is often neglected. This highlights the necessity for routine mental health assessment of these patients, which is not available in Sri Lanka. Therefore, we propose that patients with tinnitus should be routinely screened for psychiatric morbidity in Sri Lankan clinical settings. This could easily be done by ENT doctors and audiologists using appropriate screening tools and referrals made when required.

The high prevalence of depression in this study population may also be due to patients attending the otolaryngology clinics being in the severe end of the spectrum of tinnitus, as suggested by nearly half of our population reporting their tinnitus to be intolerable. Poor public awareness of this condition in Sri Lanka may explain late presentations with severe symptoms.

We found that sleep disturbance, feeling exhausted, perceived loudness of tinnitus and functional impairment were significantly associated with depression. However, this should be interpreted with caution, as except for perceieved loudeness of tinnitus, the other features are symptoms of depression. Bidirectional association is seen between nocturnal tinnitus or sleep disturbances and depression $(19,20)$. Tinnitus may keep individuals awake at night leading to depression; on the other hand insomnia associated with depression may make tinnitus more noticeable in the night. In contrast to previous studies, we found that the prevalence of depression was higher among the patients with unilateral tinnitus compared with patients with bilateral tinnitus (21).

Perhaps the most intersesting findings of our study are the factors associated with reslience in tinnitus. We found that mindfulness and ability to distract self were significantly associated with resileince, whereas optimism, religiosity, ability to see the bigger picture and perceived self-efficacy were not. Our findings contrast with previous findings of neuroticism and a lack of self assurance associated with tinnitus being related to distress, as well as the internal locus of control being associated with resilience in tinntus (3, 22-24). This has a direct implication on psychological treatment for tinnitus in Sri Lanka. It may suggest that psychotherapy for tinnitus in Sri Lanka should give more weight to mindfulness and relaxation and less weight to aspects such as cognitive restructuring.

\section{Acknowledgements}

We acknowledge the support extended by the otolaryngology surgeons Dr. S. Selvaratnam, Dr. A. Fernando, Dr. Kiridena and Dr. A. Wijerathne, and the staff of otolaryngology clinics in Kandy, Kalubowila and Matale Hospitals.

\section{Declaration of interests}

None declared.

T Abayabandara-Herath, Department of Paediatrics, Faculty of Medicine, University of Kelaniya, Ragama, Sri Lanka

A Rodrigo, Department of Psychiatry, Faculty of Medicine, University of Kelaniya, Ragama, Sri Lanka

Corresponding author: A Rodrigo

Email: asirir2000@yahoo.com

http://orcid.org/0000-0003-3962-0592

\section{References}

1. McCormack A, Edmondson-Jones M, Somerset S, Hall D. A systematic review of the reporting of tinnitus prevalence and severity. Hear Res 2016; 70(9): 337.

2. Simpson JJ, Davies WE. Recent advances in the pharmacological treatment of tinnitus. Trends Pharmacol Sci 1999; 20(1): 12-8.

3. Wallhäusser-Franke E, Delb W, Balkenhol T, et al. Tinnitus-related distress and the personality characteristic resilience. Neural Plast 2014: 6.

4. Salviati M, Bersani FS, Terlizzi S, et al. Tinnitus: clinical experience of the psychosomatic connection. NeuroPsychiatr Dis Treat 2014; 10: 267-75.

5. Pinto PC, Marcelos CM, Mezzasalma MA, Osterne FJ, de Melo Tavares de Lima MA, Nardi AE. Tinnitus and its association with psychiatric disorders: systematic review. J Laryngol Otol 2014; 128(8): 660-4.

6. Hébert S, Canlon B, Hasson D, Magnusson Hanson LL, Westerlund $\mathrm{H}$, Theorell T. Tinnitus severity is reduced with reduction of depressive mood - a prospective population study in Sweden. PLoS One 2012; 7(5): e37733. 
7. Bartels H, Pedersen SS, van der Laan BF, et al. The impact of Type D personality on health-related quality of life in tinnitus patients is mainly mediated by anxiety and depression. Otol Neurotol 2010; 31(1): 11-8.

8. Wu V, Cooke B, Eitutis S, Simpson MTW, Beyea JA. Approach to tinnitus management. Can Fam Physician 2018; 64(7): 491-5.

9. Abeysena C, Peiris U, Jayawardana P, et al. Validation of the Sinhala version of 30 - item General Health Questionnaire. International Journal of Collaborative Research on Internal Medicine and Public Health 2012; 4: 9.

10. Rodrigo A, Kuruppuarachchi KALA, Pathmeswaran, A. Validation of the Beck Depression Inventory II among the Sinhalese speaking population in Sri Lanka. SL J Psychiatry 2015; 6(2): 20-4.

11. Bonanno GA, Galea S, Bucciarelli A, et al. Psychological resilience after disaster: New York City in the aftermath of the September $11^{\text {th }}$ terrorist attack. Psychol Sci 2016; 17(3): 181-6.

12. Bonanno GA, Galea S, Bucciarelli A, et al. What predicts psychological resilience after disaster? The role of demographics, resources, and life stress. J Consult Clin Psychol 2007: 75(5); 671-82.

13. Reynolds P, Gardner D, Lee R. Tinnitus and psychological morbidity: a cross-sectional study to investigate psychological morbidity in tinnitus patients and its relationship with severity of symptoms and illness perceptions. Clin Otolaryngol Allied Sci. 2004; 29(6): 628-34.

14. Hu J, Xu J, Streelman M, Xu H, Guthrie O. The Correlation of the Tinnitus Handicap Inventory with Depression and Anxiety in Veterans with Tinnitus. Int J Otolaryngol. 2015; 2015: 689375.

15. Adoga AA, Obindo TJ. The association between tinnitus and mental illnesses. In Tech 2013; 349-69.
16. Ball HA, Siribaddana SH, Kovas Y, Glozier N, McGuffin P, Sumathipala A, Hotopf M. Epidemiology and symptomatology of depression in Sri Lanka: a crosssectional population-based survey in Colombo District. J Affect Disord 2010; 123(1-3): 188-96.

17. Weeratunga EB, Senadheera C, Ekanayake U. Psychological distress in cancer patients in Southern province of Sri Lanka. Galle Medical Journal 2016; 21(2): 1-7.

18. Herath TB, Withana M, Rodrigo C, et al. Prevalence and associations for symptoms of depression in patients with Parkinson's disease: a Sri Lankan experience. Int J Ment Health Syst. 2016; 10: 47.

19. Degeest S, Corthals P, Dhooge I, et al. The impact of tinnitus characteristics and associated variables on tinnitusrelated handicap. J Laryngol Otol 2016; 130(1): 25-31.

20. Henry JL, Wilson PH. The Psychometric Properties of Two Measures of Tinnitus Complaint and Handicap. Int Tinnitus J 1998; 4(2): 114-21.

21. Hu J, Xu J, Streelman M, Xu H, Guthrie O. The Correlation of the Tinnitus Handicap Inventory with Depression and Anxiety in Veterans with Tinnitus. Int J Otolaryngol. 2015; 2015: 689375.

22. Langguth B, Kleinjung T, Fischer B, et al. Tinnitus severity, depression, and the big five personality traits. Prog Brain Res 2007; 166: 221-5.

23. Tyler RS, Coelho C, Noble W. Tinnitus: standard of care, personality differences, genetic factors. ORL J Otorhinolaryngol Relat Spec 2006; 68(1): 14-19.

24. McCormack A, Edmondson-Jones M, Fortnum H, et al. The prevalence of tinnitus and the relationship with neuroticism in a middle-aged UK population. J Psychosom Res 2014; 76(1): 56-60.

25. Xin F, Li Q, Guan F, Suo M, Yang J, Dan Li, Zhao C. The study on psychological resilience of tinnitus and associated influencing factors. J Otology 2021; 8: 1. 